\title{
Ethylene-Inducible AP2/ERF Transcription Factor Promotes Capsaicinoid Biosynthesis in Capsicum
}

minghua deng ( $\square$ dengminghua2013@sina.com )

Yunnan Agriculture University https://orcid.org/0000-0001-8293-9035

kai zhao

Yunnan Agriculture University

junheng lv

Yunnan Agriculture University

xiang zhang

Yunnan Agriculture University

zuosen li

Yunnan Agriculture University

hong zhang

Yunnan Agriculture University

jinlong huo

Yunnan Agriculture University

hongjian wan

Zhejiang Academy of Agricultural Sciences

ziran wang

Yunnan Agriculture University

haishan zhu

Yunnan Agriculture University

xuexiao zou

Hunan Agricultural University

jinfen wen

Kunming University of Science and Technology

\section{Research Article}

Keywords: CcERF2, Capsaicinoids, Ethylene, VIGS

Posted Date: November 18th, 2021

DOI: https://doi.org/10.21203/rs.3.rs-1080827/v1 
License: (c) (i) This work is licensed under a Creative Commons Attribution 4.0 International License. Read Full License 


\section{Abstract}

Ethylene is very important in the process of plant development and regulates the biosynthesis of a lot of secondary metabolites. In these regulatory mechanisms, transcription factors that mediate ethylene signals play a very important role. Capsaicinoids (CAPs) only synthesized and accumulated in Capsicum species, causing their fruit to have a special pungent taste, which can protect against attack from herbivores and pathogens. In this study, we identified the transcription factor CCERF2, which is induced by ethylene, and demonstrated its regulatory effect on CAPs biosynthesis. Transcriptome sequencing analysis revealed the expression patterns of CCERF2 and multiple genes associated to CAPs biosynthesis were basically the same. The spatiotemporal expression results showed CCERF2 was preferentially expressed in the placenta of the spicy fruit. Ethylene can induce the expression of CCERF2 and CAPs biosynthesis genes (CBGs). CCERF2 gene silenced, 1-MCP and PZA treatments caused a decrease in expression of CBGs and a sharp decrease in content of CAPs. The results indicated CCERF2 was associated with the CAPs biosynthesis.

\section{Background}

Peppers (Capsicum spp.)are important vegetables worldwide. Pepper fruits are diverse in color, rich in nutrients, have a special pungency and aroma, and so are widely used as food additives (Ou et al, 2018). The pungency of pepper fruit is derived from capsaicinoids (CAPs), which are only biosynthesized in Capsicum plants (Iwai et al, 1979; Liu et al, 2019). The CAPs have the effect of curbing attack by herbivores and microorganisms. At the same time, CAPs are also widely used in many fields such as food, medicine, cosmetics, and agricultural pest control; CAPs are also widely used in riot prevention, personal defense, and in the military and national defense (Lejeune et al, 2007; Tewksbury et al, 2008; Ludy et al, 2012). The CAPs are synthesized and accumulated in fruit placental tissues (Iwai et al, 1979). So far, more than 22 CAPs have been identified in peppers. The most important components of CAPs are capsaicin (CaP) and dihydrocapsaicin (DhCaP). Approximately $90 \%$ of the total content of CAPs are them. The accumulation of CAPs is affected by a lot of factors, including endogenous (for example, variety and developmental stage of the fruit) and exogenous factors (for example, light, temperature, water conditions and biological stress) (Garruña-Hernández et al, 2013; Phimchan and Techawongstien 2012). The content of CAPs may vary significantly between and within pepper genotype. Genotype is the most important determinant of CAPs content (Bosland et al, 2012; Liu et al, 2017); among the five domesticated species of Capsicum, $C$. chinense and $C$. frutescens accumulated significantly higher content of CAPs (Deng et al, 2009; Bosland et al, 2012). At the same time, the content of CAPs has high plasticity, which can be affected by many complex ecological-physiological reactions and environment. It is reported that factors such as plant growth regulators, chemicals, temperature, light, and drought stress can change the content of CAPs (Gurung et al, 2011).

The CAPs are synthesized by the fusion of phenylpropane and branched-chain fatty acid pathways in the placenta (Aluru et al, 2003; Blum et al, 2003; Mazourek et al, 2009). The CAPs biosynthesis genes (CBGs) that have been determined to be involved in biosynthesis of CAPs include PAL, Ca4H, 4CL, BCAT, Kas, 
FatA, ACL, ACS, CoMT, pAMT and CS (Abraham-Juárez et al, 2008; Liu et al, 2013). The biosynthetic mechanism of CAPs has been widely elucidated using bioinformatics analysis and multi-omics (Qin et al, 2014). As CAPs have considerable application value and commercial use, a lot of effect have been performed to enhance their content. However, their biosynthesis changed largely in space/time, and expression level of CBGs is precisely regulated at the transcriptional level. Manipulating the expression level of crucial CBGs seems to be determined the encreasing content of CAPs (Abraham-Juárez et al, 2008; Sun et al, 2019; Zhu et al, 2019). In highly spicy peppers, the transcription level of CBGs (such as pAMT, Kas, and CS) is always higher than that of less spicy varieties (Abraham-Juárez et al, 2008). It is very important that manipulating the expression levels of some transcription factors (TFs) usually changes the transcription levels of all genes in the metabolic pathway, thereby affecting the content of the compound at last.

For example, the TFs CaMYB31, CaMYB108, CaMYB48, ERF and Jerf are associated with regulating the content of CAPs (Keyhaninejad et al, 2014; Phimchan et al, 2014; Arce-Rodríguez et al, 2017; Sun et al, 2019; Zhu et al, 2019). It was found that the Solanaceae-specific TF MYB31 affects the accumulation of CAPs by directly targeting CBGs resulting changing the expression level of CBGs (Zhu et al, 2019). TF CaMYB108 activates the CBG promoters, especially those of COMT, pAMT, and Kasl, and promotes enhanced expression of CBGs, thereby promoting the biosynthesis of CAPs (Sun et al, 2019). By directly binding and regulating the expression of CBGs, TF CaMYB48 participates in the biosynthesis of CAPs, but the transcriptional regulation of CAPs biosynthesis has not been fully clarified (Sun et al, 2020). Therefore, it is necessary to identify TF associated with the biosynthesis of CAPs.

Ethylene plays a significantly role in plant life process (Hu et al, 2020). After synthesis of ethylene, it binds to the receptor ETR and transmits the signal to the nucleus through MAPKK and EIN2 (Gray 2004). The EIN2 binds to EIN3/EIL1 Andthe EIN3/EIL1 bind to the ERF1 promoter. ERF1 binds to the GCC-boxcontaining genes in the downstream promoter region specifically and their expression can promote secondary metabolism product synthesis (Fujimoto et al, 2000; Brown et al, 2003; Paul et al, 2019). For example, in tobacco, ERF189 and ERF163 can bind to the GCC-box in the promoter region of the tobacco nicotine synthesis-related gene PMT2 specifically, and directly promote synthesis of tobacco alkaloids (Shoji et al, 2010). The B3 subfamily proteins ORCA3 and ORA59 of the ERF family are two typical TFs associated with the regulation of secondary metabolites. The ORCA3 can upregulate the expression level of indole alkaloid synthesis-related genes in terpenoids, and promote the terpenoid indole biosynthesis (van der Fits and Memelink 2000, 2001). Lithospermum erythrophyllum LeERF-1 affects the secondary metabolites of shikonin positively through a mechanism similar to that of ORCA3 in affecting secondary metabolites (Zhang et al, 2011). In Catharanthus roseus, CrERF5 upregulates the biosynthesis and accumulation of bisindole alkaloids (Pan et al, 2019).

The TFs and key enzymes in ethylene synthesis and its signal transduction can promote the biosynthesis of secondary metabolites. In many cases (plants, cells, and hairy roots), the addition of ethylene, addition of 1-aminocyclopropane 1-carboxylic acid (ACC), and overexpression of related TFS can increase the biosynthesis of secondary metabolites (Gantet and Memelink 2002). For example, adding $0.018 \mathrm{~mL} / \mathrm{L}$ of 
ethylene to yew cell culture can greatly increase production of taxane (Pan et al, 2000); adding ACC to Arabidopsis can promote synthesis of flavonoids (Buer et al, 2006); adding ethylene or treating cypress cells (Platycladns orientalis) with ACC can promote the biosynthesis of $\beta$-rubelin (Zhao et al, 2004); and overexpression of ORC1/ERF221 can upregulate the synthesis of pyridine alkaloids (De Boer et al, 2011).

In this paper, the ethylene-induced AP2/ERF TFs CCERF2, which was particularly expressed in the placenta was identified. We confirmed that CCERF2 was associated with the production of CAPs biosynthesis.

\section{Materials And Methods \\ 1.1 Experimental materials}

The $C$. chinense inbred line SL08 has a high CAPs content and was derived from Shuan La, the hottest pepper genotype in China (Deng et al, 2009). The $C$. annuum inbred line $\mathrm{H} 19$ has a low CAPs content, and was derived from Xiangtan Chi Ban Jiao. Pepper seeds germinated in the soil of cell plastic flats in complete darkness at $28^{\circ} \mathrm{C}$. The seedlings were grown in a greenhouse of Yunnan Agricultural University on campus under normal conditions.

\subsection{Extraction and detection of CaP and DhCaP}

The CaP and DhCaP were extracted and detected according to our method (Deng et al, 2009). The total CAPs content was calculated as $(\mathrm{CaP}+\mathrm{DhCaP}) / 0.91$. Fruits in different development stages of SL08 and $\mathrm{H} 19$ were used as material for extraction and detection of $\mathrm{CaP}$ and $\mathrm{DhCaP}$.

\subsection{RNA-sep and analysis}

RNA-sep was performed according to our method (Liu et al, 2012). Fruits of SL08 at 4, 14, 24, 34, 44, 54 days after pollination (DAP) were used to isolate total RNA.

\subsection{Exogenous substance treatments}

Fruit at 24 DAP was used to investigate the effect of different exogenous substances on expression level of CCERF2 and CBGs after $0.5,1.0,1.5,3.5,5.5,8.5$, and $11.5 \mathrm{~h}$. Treated with exogenous substances, the 24-DAP fruits of SL08 were soaked in sterile water with $0.01 \mathrm{~g} / \mathrm{L}$ 6-benzylaminopurine (6-BA), $20 \mathrm{~g} / \mathrm{L}$ PEG6000, 30\% $\mathrm{H}_{2} \mathrm{O}_{2}, 0.17 \mathrm{~g} / \mathrm{L}$ gibberellic acid $\left(\mathrm{GA}_{3}\right), 0.14 \mathrm{~g} / \mathrm{L}$ salicylic acid (SA), $17.53 \mathrm{~g} / \mathrm{L} \mathrm{NaCl}, 90$ $\mathrm{g} / \mathrm{L}$ glutamate (Glu), or $0.1 \mathrm{mmol} / \mathrm{L}$ ethephon (with sterile water as control) under normal conditions. After treatment, all fruits were frozen in liquid nitrogen and stored at $-80^{\circ} \mathrm{C}$ immediately for gene expression analysis. 


\subsection{CCERF2 cloning and bioinformatics analysis}

The open reading frame of CCERF2 was cloned according to Deng et al (2012). The primers used in the experiment are shown in Table S1. The BLAST-protein-nucleic acid (BLASTP) analysis was performed on CCERF2 through a database (www.ncbi.nlm.nih.gov); PSORT Prediction was performed for subcellular location prediction; and MEME was used to analyze the amino acid sequence motif. The Portparam tool was used to predict the physical and chemical properties of the protein. SignalP-5.0, TMHMM, and Nepos were used for signal peptide, transmembrane structure, and phosphorylation site of the protein prediction, respectively; SOPMA was used to predict its secondary structure; and ClustalX was used for sequence alignment and phylogenetic analysis. The MEGA 6.0 software was performed to construct a phylogenetic tree based on the amino acid sequence through the neighbor joining method, and the bootstrap method was used to evaluate the reliability of each node in the tree, repeated 1,000 times.

\subsection{Subcellular localization and transcriptional activation analysis}

Subcellular localization and transcriptional activation analysis were performed according to Sun et al (2020) and Zhu et al (2019). The full-length coding sequences of CCERF2 was cloned into the pAN580 (green fluorescent protein, GFP) vector and fused to the N-terminus of GFP under the control of the CaMV $35 \mathrm{~S}$ promoter. The constructs were separately introduced into tobacco protoplasts for transient expression, and the GFP fluorescence signals were detected using a Zeiss Ism710 confocal laser scanning microscope (Carl Zeiss, Inc, Jena, Germany)

\subsection{Gene expression analysis}

The RNAiso Plus (Takara, Dalian, P R China) was used to extract total RNA from pepper fruit. The synthesis of cDNA first strand refers to the instructions of the High Fidelity PrimeScript ${ }^{\circledR}$ RT-PCR Kit (Takara). According to the sequence of related genes revealed by transcriptome sequencing data, specific primers were respectively designed, and the internal reference was the $\beta$-ACTIN gene. Quantitative realtime PCR (qRT-PCR) was performed according to the method provided by the SYBR ${ }^{\circledR}$ Premix Ex TaqTM II (Tli RNaseH Plus) kit (Takara). The primers used in this study are shown in Table S1.

\subsection{VIGS analysis}

A fragment of the CCERF2 coding sequence (CDS) with low similarity to other genes was cloned into pTRV2 and generated the silencing vector pTRV2-CCERF2. The VIGS were carried as reported (Zhu et al, 2019). In short, the pTRV2-CCERF2 and pTVR1 vectors were co-injected into the cotyledon stage seedlings of line SL08. The empty vectors pTRV2 and pTVR1 were co-infiltrated as a control, and pTRV2PDS and pTVR1 were co-infiltrated as a technical control. The RNA isolated from 24-DAP fruits was used for expression analysis, and 34-DAP fruits were used for CaP and DhCaP measurement. The primer information used in this study is shown in Table S1. 
1.9 Effects of 1-methylcyclopropene (1-MCP) and Pyrazinamide (PZA) on the expression of CBGs and the content of CAPs

The SL08 pepper fruit of 24DAP was used to study the effects of 1-MCP and PZA on the expression of CBGs and the content of CAPs. 1-MCP treatment: $1 \mu \mathrm{L} \mathrm{L}-1$ 1-MCP fumigated for 12 hours and played in a dark environment with a temperature of $20 \pm 2^{\circ} \mathrm{C}$, a humidity of $85 \pm 5 \%$. PZA treatment: Spray $100 \mathrm{mM}$ PZA on the surface of the fruit until there are droplets, and played in a dark environment with a temperature of $20 \pm 2^{\circ} \mathrm{C}$, a humidity of $85 \pm 5 \%$. The fruits after 5 days of treatment were stored in the liquid nitrogen used for subsequent analysis. All experiments were repeated 3 times.

\subsection{Ethylene release rate determination}

Ethylene release rate was investigated according to the method of Xu et al (2018).

\subsection{Statistical analyses}

Each experiment contained three biological replicates and three technical replicates. The error bars mean the standard errors. One-way ANOVA was performed to identify significant differences. The relative gene expression was calculated using the $2^{-\Delta \Delta C T}$ method (Livak 2001). Without induction at the $0 \mathrm{~h}$ time point was used as control expression.

\section{Results And Analysis}

\subsection{Accumulation of CAPs}

Accumulation of $\mathrm{CaP}$ and $\mathrm{DhCaP}$ was detected in both lines, but the content of $\mathrm{CaP}$ was much higher (about 3-4 times) than that of DhCaP. The CaP and DhCaP contents were much higher in the placenta of SL08 than in H19 (Figure S1). Both CaP and DhCaP began to accumulate at 9 DAP in the placenta of SL08, reached a peak at 44 DAP, and then began to decline. Both CaP and DhCaP began to accumulate at 11 DAP in the placenta of H19, and began to decrease at 49 DAP. The CaP, DhCaP, and total CAPs contents in the placenta of SL08 were $44.4,63.1$, and 48.0 times those in $\mathrm{H} 19$, respectively.

\subsection{Expression of CCERF2 and CBGs is consistent with CAPs biosynthesis}

Expression patterns of CBGs during development stages of the placenta were analyzed using transcriptome sequencing data of the placenta and pericarp of line SL08 during the early stage of the laboratory study (Figure 1). Expressions of CCPAL, CcCa4H, Cc4CL, CcCoMT, CcPAMT, CcCS, CcBCAT, CCKAS, CCACL, CCFAT, and CCACS genes were positively associated with accumulation of CAPs in SL08. The expression of these genes (except $C C A C L$ ) showed a low expression level in fruit at 4 DAP, increased, and then decreased. Gene CCCS (Capana05g000531) was not expressed in 4-DAP fruit, and reached a 
maximum in 24-DAP fruit. Expression of CcCoMT (Capana03g001811), CATCCBCAT (Capana04g000751), CCKAS (Capana01g000111), and CCFATA (Capana06g000197) reached their highest values in 14-DAP fruit. Expression of CcCa4H (Capana06g000272) and CсрAMT(Capana10g001341) genes reached their maximum in 34-DAP fruit. Expression of CCPAL (Capana09g002199) reached its highest value in 44-DAP fruit.

Based on the transcriptome sequencing data of lines SL08 and H19 at different developmental stages of placenta completed in the laboratory, combined with the changes in the CaP and DhCaP contents of the placenta of both lines (Figure S1), the AP2/ERF TF CCERF2 (Capana01g000677) was identified. Its expression pattern was similar to that of CBGs (Figure 1). Expression of CCERF2 was almost undetectable in the early stage of placenta (4 DAP), then rose rapidly, reached a maximum at 34 DAP, and then decreased.

We measured the expression level of CCERF2 in some tissues of line SL08 and found that it was mainly expressed in the placenta (Figure 2A). It also showed considerable level of expression in seeds and pericarp, meaning that it has a role in these tissues. We also investigated CCERF2 expression in the placenta of both lines, and found significantly higher expression in SL08 than in H19 (Figure 2B). Expression of CCERF2 in the placenta has the same pattern with the content of CaP and DhCaP. Thus, our results indicated that CCERF2 may play a vital role in of CAPs biosynthesis.

\subsection{CCERF2 gene cloning and bioinformatics analysis}

Based on our transcriptome sequencing data of SL08 and the pepper genome data in the public database, specific primers were designed to clone the full-length CDS of CCERF2 from SL08. Nucleotide sequence analysis showed that CCERF2 length was $795 \mathrm{bp}$. The molecular formula of CcERF2 is $\mathrm{C}_{1328} \mathrm{H}_{2050} \mathrm{~N}_{376} \mathrm{O}_{409} \mathrm{~S}_{9}$, molecular weight is $30,115.70 \mathrm{D}$, and theoretical isoelectric point is 5.74 . It is a fatsoluble, hydrophilic, and unstable protein. CcERF2 had no signal peptide and no transmembrane structure, and is located in the nucleus ( $88.8 \%$ probability).

The protein domain prediction results showed that CCERF2 belonged to the AP2 superfamily, and the conservative amino acid sequence position was 74-131

(LYRGIRQRPWGKWAAEIRDPRKGVRVWLGTFNTAEEAARAYDKEARKIRGEKAKVNFP) (Figure 3C). This domain specifically bound to the 11-bp GCC box of the ethylene response element and was essential for the ethylene response. Based on BLAST, 48 amino acid sequences including CcERF2 were obtained, and 43 motifs were obtained after motif significance test and analysis. On the whole, the predicted motifs differed within the same family, but the conserved motifs in the same subgroup were almost the same; that is, the closer the related species, the more similar were the motifs. For peppers, they all contained 13 conserved motifs: $1-8,11,12,17,19$, and 22. Of these, motifs 1,3 , and 5 covered the AP2 conserved structure domain (Figure 3B). The secondary structure of CcERF2 protein contained 73 alpha helices (27.56\%), 22 extension chains (8.42\%), and 9 beta turns (3.41\%). There were 160 random coils $(60.61 \%)$. The evolutionary relationship of amino acid sequences showed that the selected ERF sequences were 
grouped together in different families. This meant that they were conserved in the evolutionary relationship. In genetic relationship, CcERF2 protein was the closest to Solanaceae. In the Solanaceae, it was closest to Capsicum and Solanum, and most distant to Petunia (Figure 3A). The phosphorylation sites of CcERF2 protein were predicted, and 19 amino acid residue types were screened: six types of T, three types of $Y$, and 10 types of S. CcERF2 was localized to the cell nucleus (Figure 4A) and the CcERF2 protein has strong activation activity (Figure 4B).

\subsection{CCERF2 is a TF induced by ethylene}

. Exogenous substances were applied to the 24-DAP fruit of SL08 to test the regulation model. Expression of CCERF2 in placental tissues was analyzed using qRT-PCR (Figure 5).

The fruits were treated with ethephon, which significantly induced CCERF2 expression. Compared with the control, CCERF2 expression increased by 64 times within $11.5 \mathrm{~h}$ of applying ethephon. At the same time, the effects of exogenous 6-BA, PEG, $\mathrm{H}_{2} \mathrm{O}_{2}, \mathrm{GA}_{3}, \mathrm{SA}, \mathrm{NaCl}$, and Glu on CCERF2 expression were also tested. Of these, 6-BA, $\mathrm{H}_{2} \mathrm{O}_{2}, \mathrm{GA}_{3}, \mathrm{SA}$, and $\mathrm{NaCl}$ significantly induced CCERF2 expression. Compared with the control, these exogenous substances increased CCERF2 expression; however, the modes of induced expression were not the same. In the test time range, under the 6- $\mathrm{BA}, \mathrm{H}_{2} \mathrm{O}_{2}$, and $\mathrm{NaCl}$ treatments, the CCERF2 expression showed a trend of initial increase and then decrease; however, under $\mathrm{GA}_{3}$ and $\mathrm{SA}$ treatment, CCERF2 expression showed an increasing trend. Treatment with Glu had no significant effect on CCERF2 expression and PEG treatment was inhibitory.

\subsection{Effect of ethephon on expression of CBGs}

Ethephon treatment significantly increased expression of CBGs, but the induction effect was inconsistent. Compared to controls, within $11.5 \mathrm{~h}$ of applying ethephon, expression levels of CCPAL, CCCOMT, and CCFAT were 2.3-5.0 times; of CCCa4H, CCPAmt, CCBCAT, CCACL,and CCACS were 5.8-8.3 times; of CCKAS gene was 11.1 times; and of CCCS was more than 100 times. Considering that ethephon significantly induces the expression of CCERF2, our results showed that CCERF2 was a key factor in ethylene-mediated biosynthesis of CAPs (Figure 6).

\subsection{The effect of CCERF2-silenced on CAPs content and metabolic pathways}

The VIGS vector pTRV2-CCERF2 was constructed using a vector derived from tobacco rattle virus (TRV), and a CCERF2-silenced experiment was performed. The 24-DAP fruits of SL08 were used to study. It was demonstrated that Agrobacterium infection with an empty PTRV2 vector resulted indistinctive changes in the expression level of CBGs in pepper fruits (Abraham-Juárez et al, 2008). Compared with empty vector plants, the fruits infected with the pTRV2-CCERF2 construct resulted a distinctive reduction in CCERF2 
expression (22.4\% of the empty vector) (Figure 7A). Consistent with the expression level of CCERF2, CBGs in silent plants were also significantly downregulated. In the CCERF2-silent placenta, the expression of genes derived from fatty acid metabolism pathways (such as CCKAS, CCACL, CCFAT and CCACS) only slightly changed. However, expression of genes derived from the phenylpropane pathway (such as CCPAL, CcCa4H, Cc4CL, CcComt, and CCPAmt) and CcCS underwent significant changes, and the genes whose expression was altered were consistent with the transcription level of CCERF2 (Figure 7A).

The CAPs contents of the pepper fruits infected with the empty pTRV2 vector were similar to those of uninfected plants (Abraham-Juárez et al, 2008). The 34-DAP fruits were uesed to study the effect of CCERF2 silencing on the $\mathrm{CaP}$ and DhCaP contents. Comparing with fruits infected with empty pTRV2, the $\mathrm{CaP}$ and DhCaP contents in fruits infected with pTRV2-CCERF2 were significantly reduced by $74.2 \%$ and $73.0 \%$, respectively (Figure 7B). The above results strongly supported that CCERF2 regulated certain CBGs to control CAPs biosynthesis.

\subsection{Effects of 1-MCP on CBGs expression and CAPs contents}

The effect of blocking ethylene signal transmission by 1-MCP on CBGs expression and CAPs contents had been studied. The results showed that 1-MCP treatment could remarkably reduce the expression of PAL, C4H, COMT, PAMT and CS genes (Figure 8A). 1-MCP treatment significantly reduced the content of CAPs (Figure 8B). The results indicated that the ethylene signal transduction was involved in the regulation of capsaicinoids biosynthesis.

\subsection{Effects of PZA on CBGs expression and CAPs contents}

The results indicated that after ethylene biosynthesis inhibitor PZA treated pepper fruits for $12 \mathrm{~h}$, the expression of $A C C$ oxidase-2 ( $A C O 2$ ) gene was extremely reduced (Figure $8 \mathrm{C}$ ); The endogenous ethylene release rate was conspicuouse reduced (Figure 8E); The expression of CBGs such as PAL, C4H, COMT, pAMT and CS was inhibited (Figure $8 \mathrm{C}$ ); The CAPs contents were also obviously reduced (Figure 8D). It indicated that the content of endogenous ethylene significantly affects the biosynthesis of capsaicinoids.

\section{Discussion}

Plant secondary metabolism comprises the life activity, formed in the long-term evolutionary process. The secondary metabolites are not only associated with the regulation of plant growth, but also allow plants to effectively deal with all kinds of stresses, such as fungi, pests, and herbivores (Dangl and Jones 2001). The distribution of secondary metabolites is usually specific to species, organs, and tissues; CAPs are unique synthesized in the fruit placenta of Capsicum and have obvious specificity (Mazourek et al, 2009). A variety of stress conditions (such as high temperature, hydropenia, and herbivore invasion) can induce the production of secondary metabolites, and this process is affected by a lot of plant endogenous hormones (such as ethylene). Studies have found that low temperature promotes the accumulation of CAPs in pepper fruits (Keyhaninejad et al, 2014; Arce-Rodríguez et al, 2017); drought induces biosynthesis 
of CAPs(Phimchan et al, 2014); and injury significantly increases the CAPs content of pepper fruits(ArceRodríguez et al, 2017). Plants under stress (such as high temperature, hydropenia, and herbivore attack) usually show an increase in ethylene content, which can increase the formation of secondary metabolites. Ethylene is plays a vital factor in response to all kinds of stresses (Fang et al, 2014; Hu et al, 2020). However, the effect of CAPs biosynthesis induced by ethylene needs further study.

We investigated the mechanism of ethylene on the expression of CCERF2 and CBGs. After fruits of line SL08 were treated with ethephon, expressions of CCERF2 and CBGs were significantly increased, equivalent to 2.1-110.3 times that of the control. However, the response pattern of each gene induced by ethephon slightly differed (Figure 5). A lot of studies have indicated that ethephon can upregulate the expression of multiple genes in the phenylpropane metabolic pathway, thereby promoting biosynthesis of flavonoids, anthocyanins, rutin, lignin, and procyanidins (El-Kereamy et al, 2003; Buer et al, 2006; Hossain et al, 2009; Pan et al, 2019; Paul et al, 2019; Hu et al, 2020; Zhao et al, 2020). Our experimental results are similar to those reports.

Most AP2/ERF family proteins have conserved AP2 domains, which endow them with the ability to bind to specific DNA, and have been shown to be used as regulatory factors for regulating different types of special metabolites, such as phenylpropane, betaine, glucosinolates, and analogs of carotene (Pan et al, 2019; Paul et al, 2019; Hu et al, 2020; Zhao et al, 2020). These AP2/ERF factors seem to play a similar role in regulation metabolic genes (Hu et al, 2020). In this paper, the AP2/ERF TF CCERF2, which can be used as a transcription activator to regulate CAPs biosynthesis was identified. There were 43 motifs obtained by analyzing the motif significance of the CcERF2 protein. On the whole, the predicted motifs differed within the same family, but the conserved motifs in the same subgroups were almost the same. That is, the closer the related species, the more similar were the motifs. The peppers all contained 13 conserved motifs $(1-8,11,12,17,19$, and 22$)$, of which motifs 1,3 , and 5 covered the conserved AP2 structural domain. It was reported that this type of TF can regulate the synthesis of plant secondary metabolites (Paul et al, 2019). Our results showed that the function of CcERF2 was convergent and divergent among different plant species.

Various genes related to CAPs biosynthesis have been studied (Aluru et al, 2003). However, very few TFs associated to CAPs biosynthesis have been isolated and characterized, except for some MYB TFs (ArceRodríguez et al, 2017; Sun et al, 2019; 2020; Zhu et al, 2019). We need to identify some TFs, especially AP2/ERF family TFs, which can be used as positive regulators to promote accumulation of CAPs. In the current study, based on transcription data, the AP2/ERF TF CCERF2 was selected for further analysis, because CCERF2 had a similar expression pattern to the CBGs in the transcriptome data. Silencing CCERF2 downregulated the expression of CBGs, especially the expression level of genes associated with the phenylpropane pathway, and therefore reduced its CAPs content. The results showed that CCERF2 was involved in regulating this metabolic process.

1-MCP is an ethylene receptor inhibitor (Sisler and Serek 1997; Huber 2008). It can effectively inhibit ethylene signal transduction (Du et al, 2021). A large number of studies have shown that 1-MCP can 
affect the maturation, senescence and secondary metabolism of non-atmospheric fruits by blocking ethylene signal transduction (Gómez-Lobato et al, 2012; Li et al, 2016; Zhang et al, 2018). Our research results showed that: 1-MCP significantly reduces the expression of some capsaicin biosynthesis genes, and obviously reduces the content of capsaicinoids in fruits.

PZA is a new type of ethylene biosynthesis inhibitor by inhibiting the expression of ACO2 (Sun et al, 2017). Our research found that PZA could obviously reduce the expression of $A C O 2$ in pepper fruits and remarkably reduce the production of ethylene. At the same time, it was found that PZA treatment could extremely reduce the expression of some genes related to capsaicin biosynthesis, thereby reducing the content of capsaicinoids.

\section{Declarations}

\section{Author contributions}

D.M. and Z.K. conceived and designed the study; L.J., Z.X., L.Z., Z.H., H.J., and W.H. performed the research; W.Z., Z.H., Z.X., and W.J. analyzed the data; and D.M., Z.X., and W.J. prepared the paper.

\section{Conflict of interest}

The authors declare that they have no conflicts of interest

\section{Acknowledgements}

This study was supported by the Natural Science Foundation of China (Grant No 31560556, 31160394) and the Major Science and Technology Projects in Yunnan Province (2018BB020).

\section{References}

1. Abraham-Juárez MR, Rocha-Granados MC, López MG, Rivera-Bustamante RF, Ochoa-Alejo N (2008) Virus-induced silencing of Comt, pAmt and Kas genes results in a reduction of capsaicinoid accumulation in chili pepper fruits. Planta 227: 681-695. https://doi.org/10.1007/s00425-007-0651-7

2. Aluru MR, Mazourek M, Landry LG, Curry J, Jahn M, O'Connell MA (2003) Differential expression of fatty acid synthase genes, Acl, Fat and Kas, in Capsicum fruit. J Exp Bot 54: 1655-1664. https://doi.org/10.1093/jxb/erg176

3. Arce-Rodríguez ML, Ochoa-Alejo N (2017) An R2R3-MYB transcription factor regulates capsaicinoid biosynthesis. Plant Physiol 174: 1359-1370. https://doi.org/10.1104/pp.17.00506

4. Blum E, Mazourek M, O'Connell MA, Curry J, Thorup T, Liu K, Jahn M, Paran I (2003) Molecular mapping of capsaicinoid biosynthesis genes and quantitative trait loci analysis for capsaicinoid content in Capsicum. Theor Appl Genet 108: 79-86. https://doi.org/10.1007/s00122-003-1405-y

5. Bosland PW, Coon D, Reeves G (2012) Trinidad Moruga Scorpion'pepper is the world's hottest measured chile pepper at more than two million Scoville heat units. HortTechnology 22 (4): 534-538. 
https://doi.org/10.21273/HORTTECH.22.4.534

6. Brown RL, Kazan K, McGrath KC, Maclean DJ, Manners JM (2003) A role for the GCC-box in jasmonate-mediated activation of the PDF1.2 gene of Arabidopsis. Plant Physiol 132: 1020-1032. https://doi.org/10.1104/pp.102.017814

7. Buer CS, Sukumar P, Muday GK (2006) Ethylene modulates flavonoid accumulation and gravitropic responses in roots of Arabidopsis. Plant Physiol 140: 1384-1396.

https://doi.org/10.1104/pp.105.075671

8. Dangl JL, Jones JDG (2001) Plant pathogens and integrated defence responses to infection. Nature 411: 826-833. https://doi.org/10.1038/35081161

9. De Boer K, Tilleman S, Pauwels L, Vanden Bossche R, De Sutter V, Vanderhaeghen R, Hilson P, Hamill JD, Goossens A (2011) APETALA2/ETHYLENE RESPONSE FACTOR and basic helix-loop-helix tobacco transcription factors cooperatively mediate jasmonate-elicited nicotine biosynthesis. Plant J 66: 1053-1065. https://doi.org/10.1111/j.1365-313X.2011.04566.x

10. Deng MH, Wen JF, Huo JL, Zhu HS, Wang P, Dai XZ, Zhang ZQ, Zhou H, Zou XX (2012) Molecular characterization and prokaryotic expression of orf507 sterility-associated gene in chilli pepper (Capsicum annum L.) cytoplasmic male sterility. Pak J Bot 44(5): 1497-1502. http://www.pakbs.org/pjbot/PDFs/44(5)/02.pdf

11. Deng MH, Wen JF, Zhu HS, Zou XX (2009) The hottest pepepr variety in China. Genet Resour Crop Evol 56: 605-608. https://doi.org/ 10.1007/s10722-009-9445-z

12. Du Y, Jin T, Zhao H, Han C, Sun F, Chen Q, Yue F, Luo Z, Fu M (2021) Synergistic inhibitory effect of 1methylcyclopropene (1-MCP) and chlorine dioxide (ClO2) treatment on chlorophyll degradation of green pepper fruit during storage. Postharvest Biol Technol 171: 111363. https://doi.org/ 10.1016/j.postharvbio.2020.111363

13. El-Kereamy A, Chervin C, Roustan JP, Cheynier V, Souquet JM, Moutounet M, Raynal J, Ford C, Latche A, Pech JC, Bouzayen M (2003) Exogenous ethylene stimulates the long-term expression of genes related to anthocyanin biosynthesis in grape berries. Physiol Plant 119: 175-182. https://doi.org/10.1034/j.1399-3054.2003.00165.x

14. Fang RJ, Zhao H, Liao RH, Tang CY, Wu FY, Zhu Y, Pang YJ, Lu GH, Wang XM, Yang RW, Qi JL, Yang $\mathrm{YH}$ (2014) Dual regulating effects of ethylene on the formation of plant secondary metabolites. Chin Bull Bot 49: 626-639. https://doi.org/ 10.3724/SP.J.1259.2014.00626

15. Fujimoto SY, Ohta M, Usui A, Shinshi H, Ohme-Takagi M (2000) Arabidopsis ethylene-responsive element binding factors act as transcriptional activators or repressors of GCC box-mediated gene expression. Plant Cell 12: 393-404. https://doi.org/ 10.2307/3870944

16. Gantet P, Memelink J (2002) Transcription factors: tools to engineer the production of pharmacologically active plant metabolites. Trends Pharmacol Sci 23: 563-569. https://doi.org/ 10.1016/S0165-6147(02)02098-9

17. Garruña-Hernández R, Monforte-González M, Canto-Aguilar A, Vázquez Flota F, Orellana R (2013) Enrichment of carbon dioxide in the atmosphere increases the capsaicinoids content in Habanero 
peppers (Capsicum chinense Jacq.). J Agr Food Chem 93: 1385-1388. https://doi.org/ 10.1002/jsfa.5904

18. Gómez-Lobato ME, Hasperue JH, Civello PM, Chaves AR, Martínez GA (2012) Effect of 1-MCP on the expression of chlorophyll degrading genes during senescence of broccoli (Brassica oleracea L.). Sci Hortic 144: 208-211.

19. Gray WM, (2004). Hormonal regulation of plant growth and development. PLoS Biol 2: E311. https://doi.org/ 10.1016/j.scienta.2012.07.017

20. Gurung T, Techawongstien S, Suriharn B, Techawongstien S (2011) Impact of environments on the accumulation of capsaicinoids in Capsicum spp.. HortScience 46: 1-6. https://doi.org/ $10.2514 / 1.16041$

21. Hossain MA, Kim S, Kim KH, Lee SJ, Lee H (2009) Flavonoid compounds are enriched in lemon balm (Melissa officinalis) leaves by a high level of sucrose and confer increased antioxidant activity. Hortscience 44: 1907-1913. https://doi.org/ 10.1007/s10658-009-9505-4

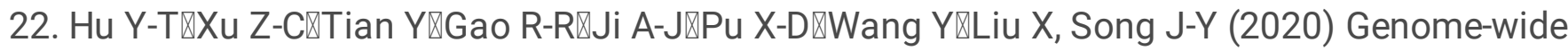
identification and analysis of AP2/ERF transcription factors related to camptothecin biosynthesis in Camptotheca acuminate. Chin J Nat Med (08): 582-593. https://doi.org/ 10.1016/S18755364(20)30070-4

23. Huber D J (2008) Suppression of ethylene responses through application of 1- methylcyclopropene: a powerful tool for elucidating ripening and senescence mechanisms in climacteric and nonclimacteric fruits and vegetables. HortScience 43: 106-111. https://doi.org/10.1007/s10658007-9208-7

24. Iwai K, Suzuki T, Fujiwake H (1979) Formation and accumulation of pungent principle of hot pepper fruits, capsaicin and its analogues, in Capsicum annuun var. annuun cv. karayatsubusa at different growth stages after flowering. Agric Biol Chem 43: 2493-2498. https://doi.org/ 10.1080/00021369.1979.10863843

25. Keyhaninejad N, Curry J, Romero J, O'Connell MA (2014) Fruit specific variability in capsaicinoid accumulation and transcription of structural and regulatory genes in Capsicum fruit. Plant Sci (215216): 59-68. https://doi.org/ 10.1016/j.plantsci.2013.10.013

26. Lejeune MPGM, Kovacs EMR, Westerterp-Plantenga MS (2007) Effect of capsaicin on substrate oxidation and weight maintenance after modest bodyweight loss in human subjects. Brit J Nutr 90 : 651-659. https:// doi.org/ 10.1079/BJN2003938

27. Li L, Lichter A, Chalupowicz D, Gamrasni D, Goldberg T, Nerya O, Ben-Arie R, Porat R (2016) Effects of the ethylene-action inhibitor 1-methylcyclopropene on postharvest quality of non-climacteric fruit crops. Postharvest Biol Technol 111: 322-329. https://doi.org/ 10.1016/j.postharvbio.2015.09.031

28. Liu F, Yu H Deng Y, Zheng J, Liu M, Ou L, Yang B, Dai X, Ma Y, Feng S, He S, Li X, Zhang Z, Chen W, Zhou S, Chen R, Liu M, Yang S, Wei R, Li H, Li F, Ouyang B, Zou X (2017) PepperHub, an informatics hub for the chili pepper research community. Mol Plant 10: 1129-1132. https://doi.org/ 10.1016/j.molp.2017.03.005 
29. Liu S, Li W, Wu Y, Chen C, Lei J (2013) De novo transcriptome assembly in chili pepper (Capsicum frutescens) to identify genes involved in the biosynthesis of capsaicinoids. PLoS ONE 8: e48156. https://doi.org/ 10.1371/journal.pone.0048156

30. Liu SQ, Chen CM, Chen GJ, Cao BH, Chen QH, Lei JJ (2012) RNA-Sequencing tag profiling of the placenta and pericarp of pungent pepper provides robust candidates, contributing to capsaicinoid biosynthesis. Plant Cell, Tiss \& Org Cult 110(1): 111-121. https://doi.org/ 10.1007/s11240-012-01358

31. Liu Y, Lv J, Liu Z, Wang J, Yang B, Chen W, Ou L, Dai X, Zhang Z, Zou X (2019) Integrative analysis of metabolome and transcriptome reveals the mechanism of color formation in pepper fruit (Capsicum annuum L.). Food Chem 125629. https://doi.org/ 10.1016/j.foodchem.2019.125629

32. Livak KJ, Schmittgen TD (2001) Analysis of relative gene expression data using real-time quantitative PCR and the 2(-Delta Delta C(T)) Method. Methods 25: 402-408. https://doi.org/ $10.1006 /$ meth.2001

33. Ludy MJ, Moore GE, Mattes RD (2012) The effects of capsaicin and capsiate on energy balance: critical review and meta-analyses of studies in humans. Chem Senses 37: 103-121. https://doi.org/ 10.1093/chemse/bjr100

34. Mazourek M, Pujar A, Borovsky Y, Paran I, Mueller L, Jahn MM (2009) A dynamic interface for capsaicinoid symstems biology. Plant Physiol 150: 1806-821. https://doi.org/ 10.1104/pp.109.136549

35. Ou L, Li D, Lv J, Chen W, Zhang Z, Li X, Yang B, Zhou S, Yang S, Li W, Gao H, Qin Z, Yu H, Ouyang B, Li F, Liu F, Zheng J, Liu Y, Wang J, Wang B, Dai X, Ma Y, Zou X (2018) Pan-genome of cultivated pepper (Capsicum) and its use in gene presence-absence variation analyses. New phytol 220(2): 360. https://doi.org/ 10.1111/nph.15413

36. Pan Q, Wang C, Xiong Z, Wang H, Fu X, Shen Q, Peng B, Ma Y, Sun X, Tang K (2019) CrERF5, an AP2/ERF transcription factor, positively regulates the biosynthesis of bisindole alkaloids and their precursors in Catharanthus roseus. Front Plant Sci 10: 931. https://doi.org/ 10.3389/fpls.2019.00931

37. Pan ZW, Wang HQ, Zhong JJ (2000) Scale-up study on suspension cultures of Taxus chinensis cells for production of taxane diterpene. Enzyme Microb Technol 27: 714-723. https://doi.org/ 10.1016/S0141-0229(00)00276-3

38. Paul P, Singh SK, Patra B, Liu X, Pattanaik S, Yuan L (2020) Mutually regulated AP2/ERF gene clusters modulate biosynthesis of specialized metabolites in plants. Plant physiol 182(2): 840-856. https://doi.org/ 10.1104/pp.19.00772

39. Phimchan P, Chanthai S, Bosland PW, Techawongstien S (2014) Enzymatic changes in phenylalanine ammonia-lyase, cinnamic-4-hydroxylase, capsaicin synthase, and peroxidase activities in Capsicum under drought stress. J Agric Food Chem 62: 7057-7062. https://doi.org/ 10.1021/jf4051717

40. Phimchan P, Techawongstien S (2012) Impact of drought stress on the accumulation of capsaicinoids in Capsicum cultivars with different initial capsaicinoid levels. HortScience 47(9): 
1204-1209. https://doi.org/ 10.21273/HORTSCI.47.9.1204

41. Qin C, Yu C, Shen Y, Fang X, Chen L, Min J, Cheng J, Zhao S, Xu M, Luo Y, Yang Y, Wu Z, Mao L, Wu H, LingHu C, Zhou H, Lin H, Gonzalez-Morales S, Trejo-Saavedra DL, Tian H, Tang X, Zhao M, Huang Z, Zhou A, Yao X, Cui J, Li W, Chen Z, Feng Y, Niu Y, Bi S, Yang X, Li W, Cai H, Luo X, Montes-Hernandez S, Leyva-Gonzalez MA, Xiong Z, He X, Bai L, Tan S, Tang X, Liu D, Liu J, Zhang S, Chen M, Zhang L, Zhang L, Zhang Y, Liao W, Zhang Y, Wang M, Lv X, Wen B, Liu H, Luan H, Zhang Y, Yang S, Wang X, Xu J, Li X, Li S, Wang J, Palloix A, Bosland PW, Li Y, Krogh A, Rivera-Bustamante RF, Herrera-Estrella L, Yin Y, Yu J, Hu K, Zhang Z (2014) Whole-genome sequencing of cultivated and wild peppers provides insights into Capsicum domestication and specialization. Pro Natl Acad Sci USA 111: 5135-5140. https://doi.org/ 10.1073/pnas.1400975111

42. Shoji T, Kajikawa M, Hashimoto $T$ (2010) Clustered transcription factor genes regulate nicotine biosynthesis in tobacco. Plant Cell 22: 3390-3409. https://doi.org/ 10.1105/tpc.110.078543

43. Sisler EC, Serek M (1997) Inhibitors of ethylene responses in plants at the receptor level: recent developments. Physiol Plant 100: 577-582. https://doi.org/ 10.1034/j.1399-3054.1997.1000320.x

44. Sun B, Zhu Z, Chen C, Chen G, Cao B, Chen C, Lei J (2019) Jasmonate-inducible R2R3-MYB transcription factor regulates capsaicinoid biosynthesis and stamen development in Capsicum. J Agr Food Chem 67: 10891-10903. https://doi.org/ 10.1021/acs.jafc.9b04978

45. Sun B, Zhou X, Chen C, Chen C, Chen K, Chen M, Liu S, Chen G, Cao B, Cao F, Lei J, Zhu Z (2020) Coexpression network analysis reveals an MYB transcriptional activator involved in capsaicinoid biosynthesis in hot peppers. Hortic Res 7: 162. https://doi.org/ 10.1038/s41438-020-00381-2

46. Sun X, Li Y, He W, Ji C, Xia P, Wang Y, Du S, Li H, Raikhel N, Xiao J, Guo H (2017) Pyrazinamide and derivatives block ethylene biosynthesis by inhibiting ACC oxidase. Nature Comm 8:

15758. https://doi.org/ 10.1038/ncomms 15758

47. Tewksbury JJ, Reagan KM, Machnicki NJ, Carlo TA, Haak DC, Peñaloza LC, Levey DJ (2008) Evolutionary ecology of pungency in wild chilies. Pro Natl Acad Sci USA 105: 11808-11811. https://doi.org/ 10.1073/pnas.0802691105

48. van der Fits L, Memelink J (2000) ORCA3, a jasmonateresponsive transcriptional regulator of plant primary and secondary metabolism. Science 289: 295-297. https://doi.org/

10.1126/science.289.5477.295

49. van der Fits L, Memelink J (2001) The jasmonateinducible AP2/ERF-domain transcription factor ORCA3 activates gene expression via interaction with a jasmonate-responsive promoter element. Plant J 25: 43-53. https://doi.org/ 10.1111/j.1365-313X.2001.00932.x

50. Xu L, Yue Q, Xiang G, Bian F, Yao Y (2018) Melatonin promotes ripening of grape berry via increasing the levels of $A B A, \mathrm{H}_{2} \mathrm{O}_{2}$, and particularly ethylene. Hortic Res 5: 1-11. https://doi.org/ 10.1038/s41438-018-0045-y

51. Zhang W, Zou A, Miao J, Yin Y, Tian R, Pang Y, Yang R, Qi J, Yang Y (2011) LeERF-1, a novel AP2/ERF family gene within the B3 subcluster, is down-regulated by light signals in Lithospermum erythrorhizon. Plant Biol 13: 343-348. https://doi.org/ 10.1111/j.1438-8677.2010.00375.x

Page $16 / 23$ 
52. Zhang XM, Li JP, Fu MR, Jin T, Yang XY, Han C, Chen QM, Yue FL (2018) Effects of the combination of 1-MCP and $\mathrm{ClO}_{2}$ on shelf life quality of green pepper. Sci Technol Food Ind 39: 275280. https://doi.org/ 10.13386/j.issn1002-0306.2018.13.050

53. Zhao C, Liu X, Gong Q, Cao J, Shen W, Yin X, Grierson D, Zhang B, Xu C, Li X, Chen K, Sun C (2020) Three AP2/ERF family members modulate flavonoid synthesis by regulating type IV chalcone isomerase in citrus. Plant Biotechnol J 1-18. https://doi.org/ 10.1111/pbi.13494

54. Zhao J, Zheng SH, Fujita K, Sakai K (2004) Jasmonate and ethylene signaling and their interaction are integral parts of the elicitor signaling pathway leading to $\beta$-thujaplicin biosynthesis in Cupressus Iusitanica cell cultures. J Exp Bot 55: 1003-1012. https://doi.org/ 10.1093/jxb/erh127

55. Zhu Z, Sun B, Cai W, Zhou X, Mao Y, Chen C, Wei J, Cao B, Chen C, Chen G, Lei J (2019) Natural variations in the MYB transcription factor MYB31 determine the evolution of extremely pungent peppers. New Phytol 223: 922-938. https://doi.org/ info:doi/10.1111/nph.15853

\section{Figures}

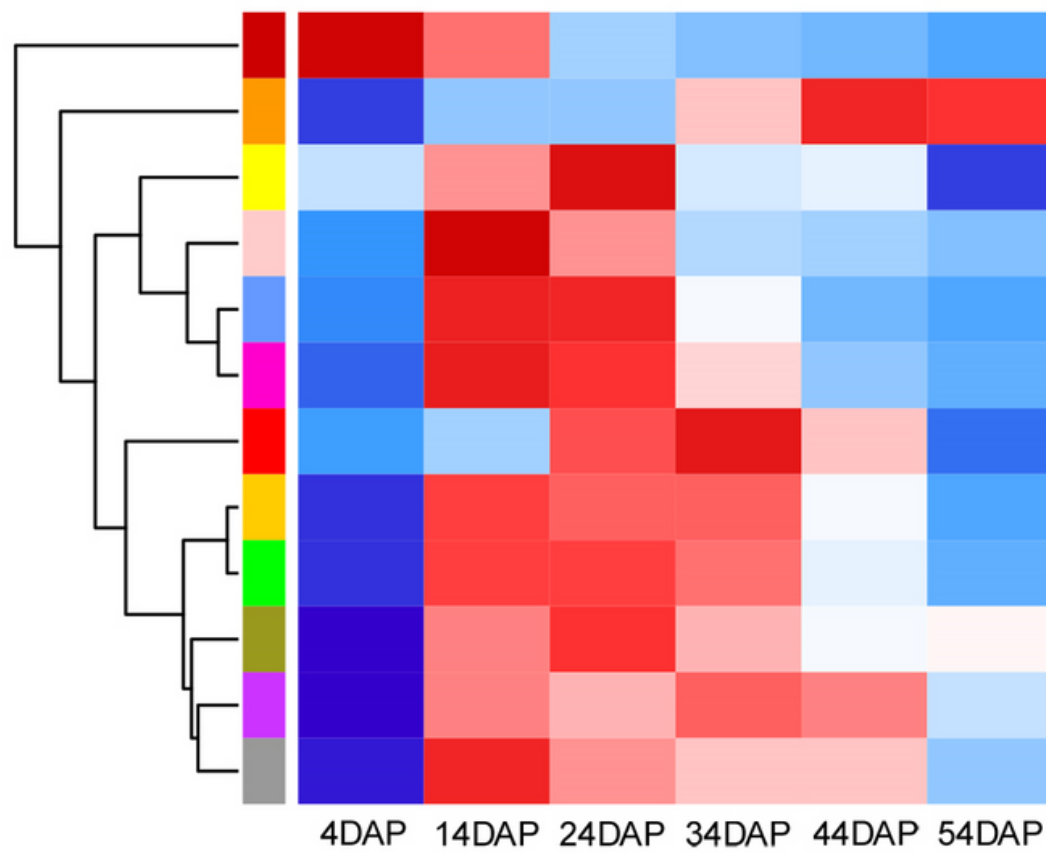

\begin{tabular}{|c|c|c|}
\hline ACL & \multicolumn{2}{|c|}{ Gene ID } \\
\hline PAL & 1.5 & Capana01g000677 \\
\hline is & 1 & Capana09g002199 \\
\hline ATA & & Capana06g000272 \\
\hline MT & 0.0 & Capana06g001083 \\
\hline & 0 & Capana03g001811 \\
\hline RF2 & -0.5 & Capana10g001341 \\
\hline $4 \mathrm{H}$ & & Capana05g000531 \\
\hline & & Capana04g000751 \\
\hline & -1.5 & Capana01g000111 \\
\hline & & Capana03g000656 \\
\hline & & Capana06g000197 \\
\hline & & Capana02g003677 \\
\hline
\end{tabular}

\section{Figure 1}

Heat map indicatingthe CBGs and CCERF2 expression patterns in fruit of inbred line SL08. The heatmap was generated by transcriptome sequencing data with $\mathrm{R}$ software packages. The data were normalized in 
each row, and the numbers on the right side of the figure indicate the gene expression level corresponding to color.
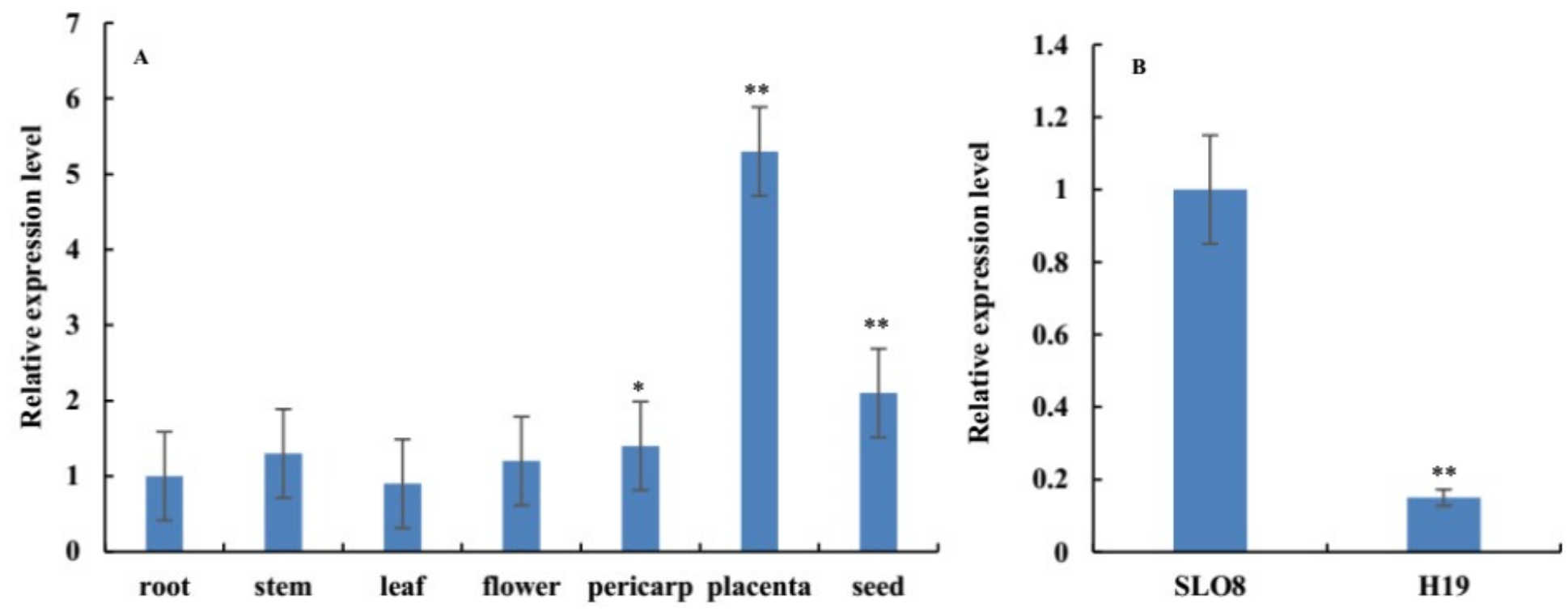

Figure 2

Differential expression assays of CcERF2 (A) Differential expression assays of CcERF2 in different tissues of pepper inbred line SL08; Roots, stems, leaves and flowers were collected from 30-day-old seedlings. Fruits at 24 DAP was collected and was divided into pericarps, placentas and seeds. The relative expression of the root was set to 1 , and that of all the other tissues was measured relative to that of the root. (B) Differential expression assays of CcERF2 in pepper inbred line SL08 and H19 fruits. Fruits at 24 DAP was collected to investigate the CCERF2 expression. The relative expression of the SL08 was set to 1 , and that of $\mathrm{H} 19$ was measured relative to that of the SL08. The experiments were replicated three biological times and three technical times. Data are expressed as the mean $\pm S D(n=9)$. Student's $t$ test was used to identify significant differences compared to the control $\left(* P<0.05\right.$, $\left.{ }^{*} P<0.01\right)$.
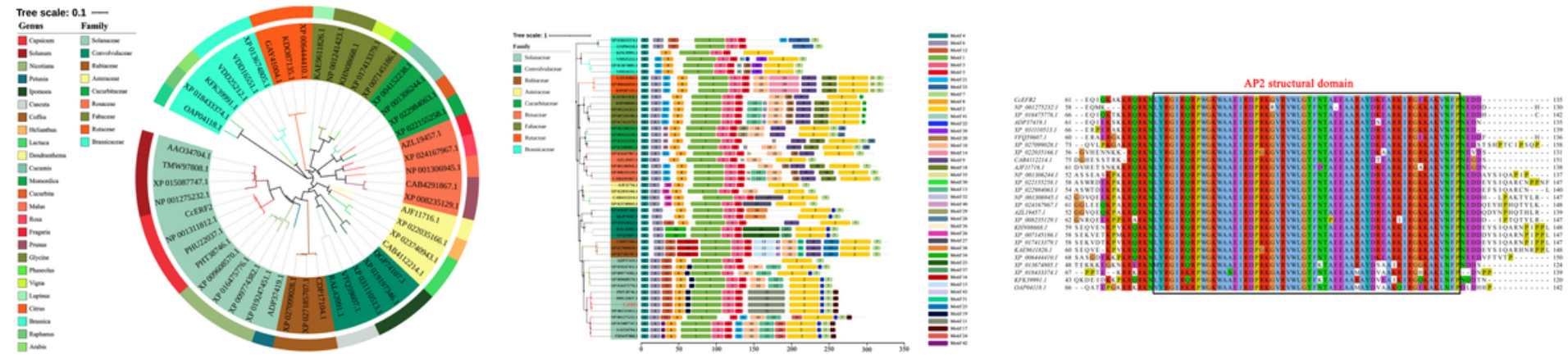

\section{Figure 3}

Phylogenic tree of ERF TFs, analysis of promoter cis-acting elements and multiple amino acid sequence alignment of CcERF2 TFs. (A) Phylogenetic tree comprising CcERF2 in C. chinense and 47 ERF2 TFs in plant. (B) Promoter cis-acting elements comprising CcERF2 in C. chinense and 47 ERF2 promoters in plant. (C) Multiple amino acid sequence alignment of ERF2 TFs. 


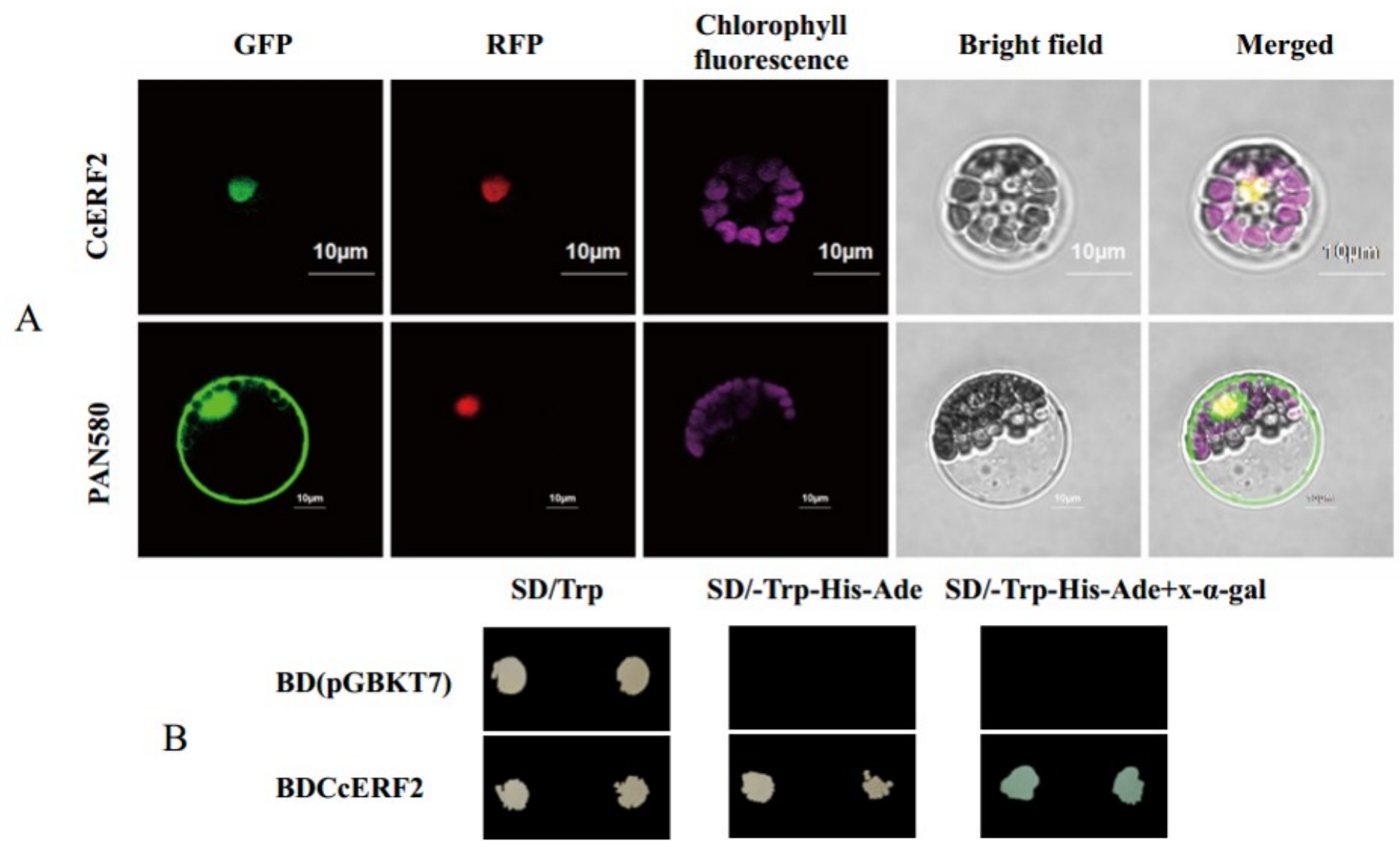

Figure 4

Subcellular localization and transcriptional activation analysis (A) GFP signals indicated that CcERF2 was localized to nucleus. GFP fluorescence signals were detected using a Zeiss Ism710 confocal laser scanning microscope (Carl Zeiss, Inc., Jena, Germany). Scale bars: $10 \mu \mathrm{m}$. (B) Auxotroph plates of SD/Leu-His-Ade (middle) and SD/-Leu-His-Ade-x-a-gal (right) showing transcriptional activation of protein. SD/Trp, medium lacking tryptophan; SD/-Trp-His-Ade, medium lacking tryptophan, histidine and adenine. 


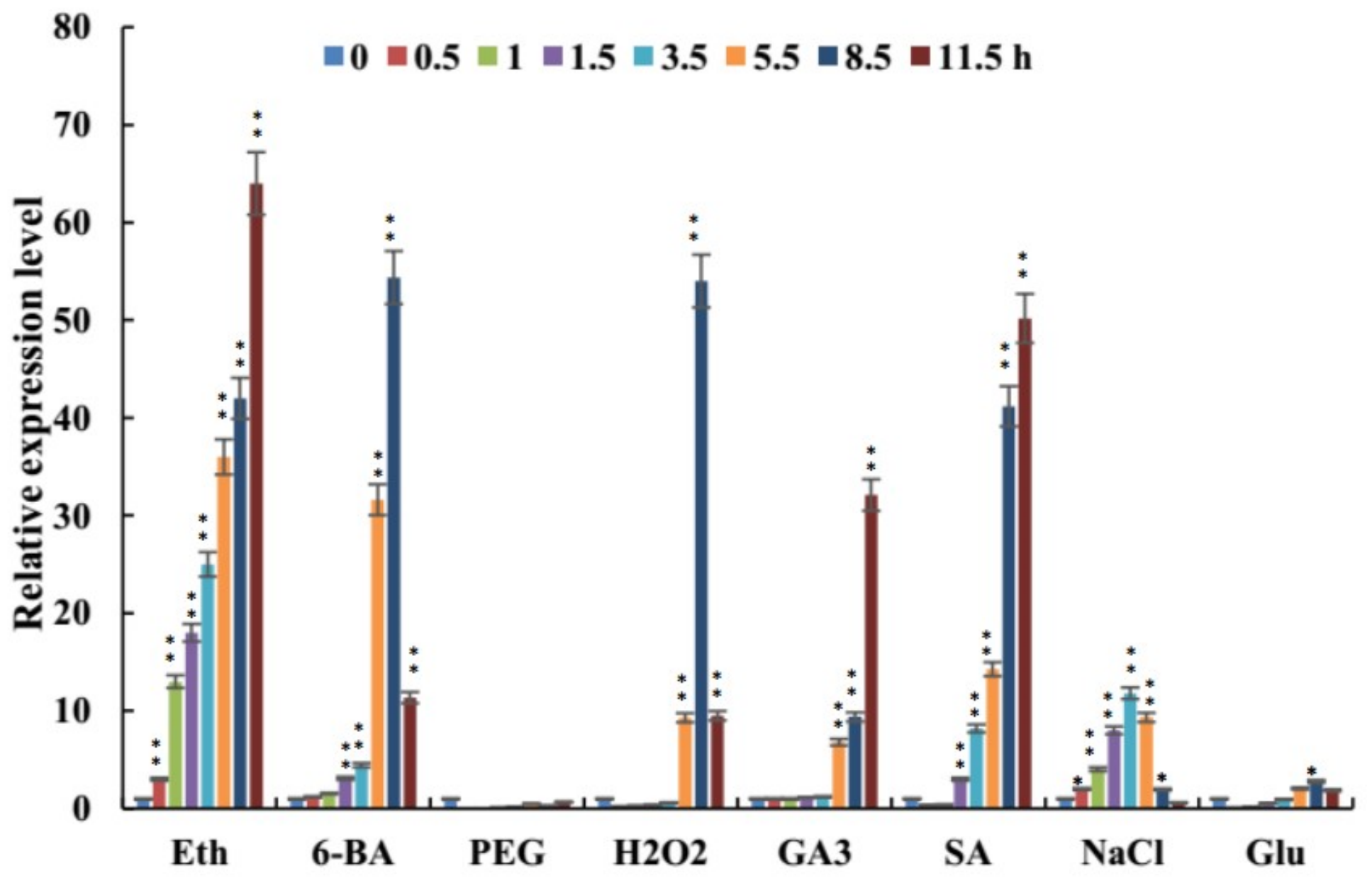

Figure 5

Effect of elicitors (Eth, 6-BA, PEG, H2O2, GA3, SA, NaCl and Glu) on CcERF2 expression. The 24 DAP pepper fruits were treated with elicitors (Eth, 6-BA, PEG, $\mathrm{H} 2 \mathrm{O} 2, \mathrm{GA} 3, \mathrm{SA}, \mathrm{NaCl}$ or Glu), and the fruit were sampled after elicitation for $0.5,1.0,1.5,3.5,5.5,8.5$, and $11.5 \mathrm{~h}$. Without induction at the $0 \mathrm{~h}$ time point was used as control expression. The experiments were replicated three biological times and three technical times. Data are expressed as the mean $\pm S D(n=9)$. Student's $t$ test was used to identify significant differences compared to the control $(* P<0.05$, **P $<0.01)$. 

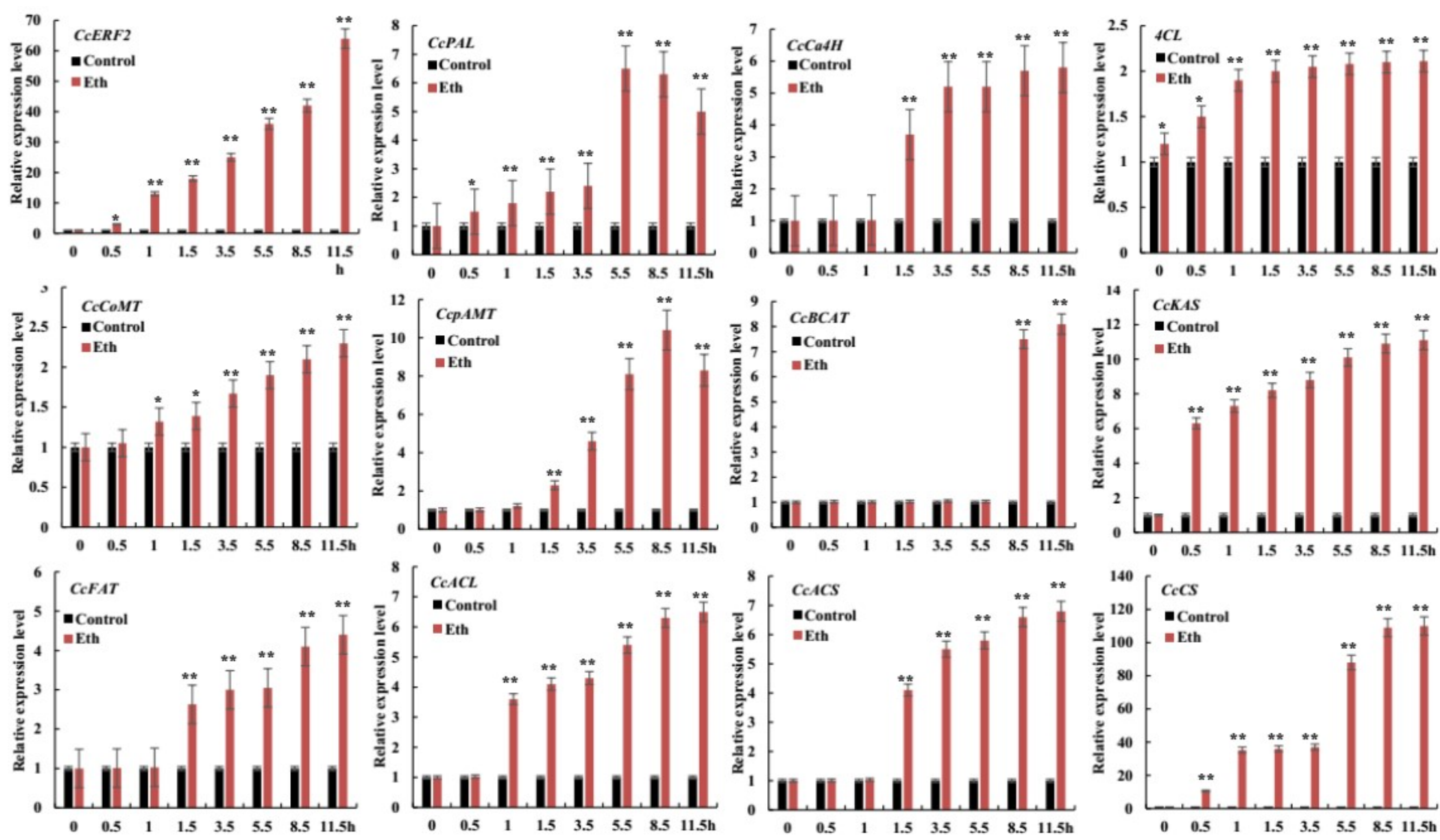

Figure 6

Expression profiles of CBGs after treatment with Eth. The 24 DAP pepper fruits were treated with Eth, and the fruit were sampled after elicitation for $0.5,1.0,1.5,3.5,5.5,8.5$, and $11.5 \mathrm{~h}$. Without induction at the 0 $\mathrm{h}$ time point was used as control expression. The experiments were replicated three biological times and three technical times. Data are expressed as the mean $\pm S D(n=9)$. Student's $t$ test was used to identify significant differences compared to the control ( ${ }^{*}<<0.05$, $\left.{ }^{*} \mathrm{P}<0.01\right)$.
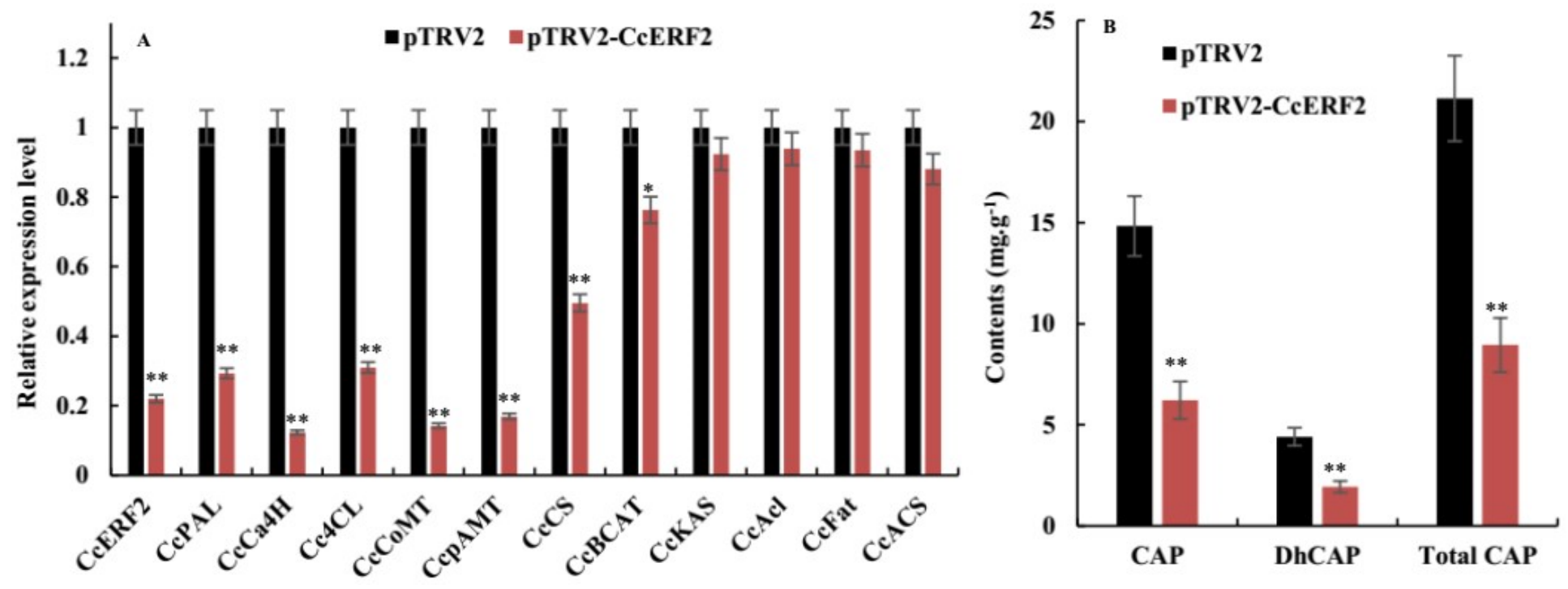

Figure 7 
Expression level of CBGs and CcERF2, relative contents of CaP, DhCaP and CAPs in the control (empty vector) and CcERF2-silenced fruit. (A) Expression level of CBGs and CcERF2 in the control (empty vector) and CCERF2-silenced fruit. Fruits at 24 DAP was sampled to perform the expression level of CBGs and CcERF2. The relative expression level of the CBGs and CCERF2 in the control were set to 1, and that of in the CcERF2-silenced fruit were measured relative to that in the control. (B) Relative contents of CaP, DhCaP and CAPs in the control (empty vector) and CcERF2-silenced fruit at 34 DAP. The experiments were replicated three biological times and three technical times. Data are expressed as the mean \pm SD ( $n$ $=9)$. Student's $t$ test was used to identify significant differences compared to the control $\left({ }^{\star} P<0.05,{ }^{\star *} P<\right.$ $0.01)$.
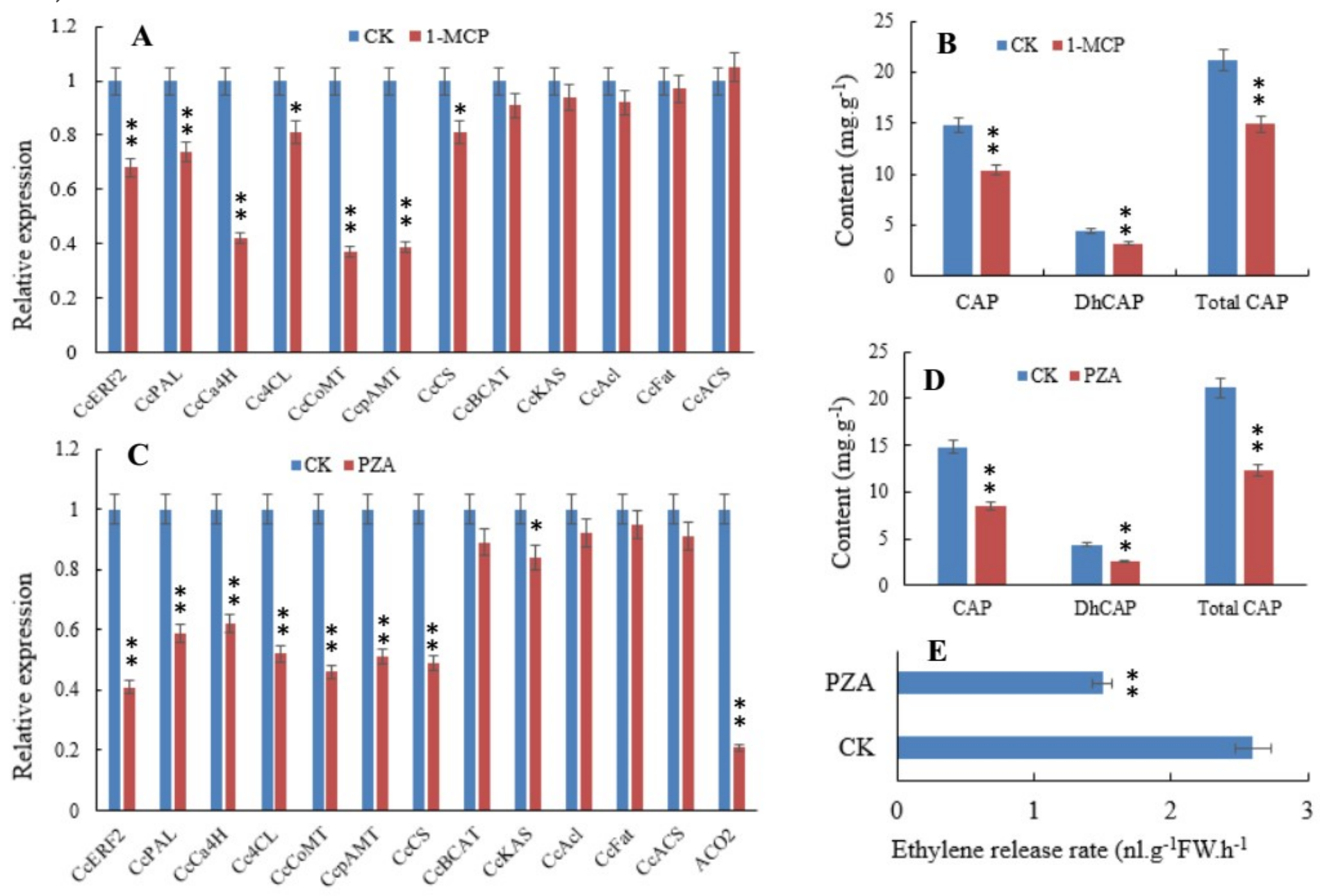

Ethylene release rate $\left(\mathrm{nl} \cdot \mathrm{g}^{-1} \mathrm{FW} \cdot \mathrm{h}^{-1}\right.$

\section{Figure 8}

Expression level of CBGs and CcERF2, relative contents of CaP, DhCaP and CAPs in 1-MCP and PZA treatment fruit. (A) Expression level of CBGs and CCERF2 in the 1-MCP treatment fruit. Fruits at 24 DAP was sampled to perform the expression level of CBGs and CCERF2. The relative expression level of the CBGs and CCERF2 in the control were set to 1, and that of in 1-MCP treatment fruit were measured relative to that in the control. (B) Relative contents of CaP, DhCaP and CAPs in the 1-MCP treatment fruit at 29 DAP. (C) Expression level of CBGs, CcERF2 and ACO2 in the PZA treatment fruit. Fruits at 24 DAP was sampled to perform the expression level of CBGs and CcERF2. The relative expression level of the CBGs and CCERF2 in the control were set to 1, and that of in PZA treatment fruit were measured relative to that 
in the control. (D) Relative contents of CaP, DhCaP and CAPs in the PZA treatment fruit at 29 DAP. (E) Ethylene release rate in the PZA treatment fruit at 29 DAP. The experiments were replicated three biological times and three technical times. Data are expressed as the mean $\pm S D(n=9)$. Student's $t$ test was used to identify significant differences compared to the control $\left(* P<0.05\right.$, $\left.{ }^{*} P<0.01\right)$.

\section{Supplementary Files}

This is a list of supplementary files associated with this preprint. Click to download.

- FigureS1.jpg

- TableS1.docx 